\title{
Full-arch accuracy of five intraoral scanners: In vivo analysis of trueness and precision
}

\author{
Miran Kwon ${ }^{\mathrm{a}}$ \\ Youngmok $\mathrm{Cho}^{\mathrm{b}}$ \\ Dong-Wook Kim ${ }^{\mathrm{a}}$ \\ MyungSu Kim ${ }^{\mathrm{a}}$ \\ Yoon-Ji Kim ${ }^{\mathrm{c}}$ (1) \\ Minho Chang ${ }^{b}$
}

${ }^{a}$ Department of Orthodontics, Korea University Graduate School of Clinical Dentistry, Seoul, Korea

${ }^{b}$ Department of Mechanical Engineering, Korea University School of Engineering, Seoul, Korea

'Department of Orthodontics, Asan Medical Center, University of Ulsan College of Medicine, Seoul, Korea

\begin{abstract}
Objective: To evaluate the trueness and precision of full-arch scans acquired using five intraoral scanners and investigate the factors associated with the dimensional accuracy of the intraoral scan data. Methods: Nine adult participants (mean age, $34.3 \pm 8.3$ years) were recruited. Four zirconium spheres (Ø $6 \mathrm{~mm}$ ) were bonded to the canines and the molars. Following acquisition of reference scans using an industrial-grade scanner, five intraoral scanners, namely i500, CS3600, Trios 3, iTero, and CEREC Omnicam, were used to scan the arches. Linear distances between the four reference spheres were automatically calculated, and linear mixed model analysis was performed to compare the trueness and precision of the intraoral scan data among the different scanners. Results: The absolute mean trueness and precision values for all intraoral scanners were $76.6 \pm 79.3$ and $56.6 \pm 52.4 \mu \mathrm{m}$, respectively. The type of scanner and the measured linear distances had significant effects on the accuracy of the intraoral scan data. With regard to trueness, errors in the intermolar dimension and the distance from the canine to the contralateral molar were greater with Omnicam than with the other scanners. With regard to precision, the error in the linear distance from the canine to the molar in the same quadrant was greater with Omnicam and CS3600 than with the other scanners. Conclusions: The dimensional accuracy of intraoral scan data may differ significantly according to the type of scanner, with the amount of error in terms of trueness being clinically significant.
\end{abstract}

[Korean J Orthod 2021;51(2):95-104]

Key words: Digital impression, Intraoral scanner, Precision, Trueness

Received August 25, 2020; Revised October 31, 2020; Accepted November 2, 2020.

Corresponding author: Yoon-Ji Kim.

Assistant Professor, Department of Orthodontics, Asan Medical Center, University of Ulsan School of Medicine, 88 Olympic-ro 43-gil, Songpa-gu, Seoul 05505, Korea.

Tel +82-2-3010-3845 e-mail yn0331@gmail.com

How to cite this article: Kwon M, Cho Y, Kim DW, Kim MS, Kim YJ, Chang M. Full-arch accuracy of five intraoral scanners: In vivo analysis of trueness and precision. Korean $\mathrm{J}$ Orthod 2021;51:95-104.

(C) 2021 The Korean Association of Orthodontists.

This is an Open Access article distributed under the terms of the Creative Commons Attribution Non-Commercial License (http://creativecommons.org/licenses/by-nc/4.0) which permits unrestricted non-commercial use, distribution, and reproduction in any medium, provided the original work is properly cited. 


\section{INTRODUCTION}

The accuracy of digital impressions obtained using intraoral scanners for a single tooth is equivalent or even superior to that of conventional impressions. ${ }^{1-3}$ However, the dimensional accuracy of full-arch intraoral scan data acquired using an intraoral scanner, relative to the accuracy of conventional impressions, remains controversial. ${ }^{1,45}$

Because of the limited field of view provided by intraoral scanners, several small, three-dimensional (3D) scan data are acquired and superimposed to create the final 3D surface data. However, errors may occur during the process of integrating the numerous acquired images. When larger objects are scanned, the amount of error may increase because a larger number of raw images need to be processed, which results in dimensional inaccuracies. A full-arch scan starts from the most distal molar and ends at the most distal molar on the contralateral side; therefore, errors in superimposition accumulate, leading to deformation of the arch shape and errors in the arch dimensions, particularly in the molar region. ${ }^{6-8}$ The dark and humid oral cavity with anatomic structures such as the tongue and buccal mucosa also contribute to inaccuracies in the scan data. ${ }^{9}$

Many studies have evaluated the accuracy of fullarch scans obtained using intraoral scanners, although most have conducted in vitro evaluations using dental casts. Errors in intraoral scan data may be greater when a patient is scanned than when a dental cast is scanned, considering the transparency of natural teeth and optical reflections as well as the humid intraoral environment. ${ }^{10}$ Only a few studies have evaluated fullarch scans acquired directly in a patient's oral cavity. ${ }^{11}$ Acquisition of an accurate reference data set for in vivo analysis of trueness is difficult because dental arches cannot be scanned with tactile or other high-precision optical laboratory scanners. Previous in vivo studies used gypsum casts obtained from conventional impressions as a reference or only measured the precision of intra- oral scan data. ${ }^{12-14}$ The dimensional accuracy of full-arch scans obtained using industrial scanners is reportedly higher than that of conventional impressions. ${ }^{15}$ Therefore, it may be used as a reference for measuring the precision and trueness of full-arch impressions. In one study, the researchers obtained a direct scan of the teeth using an industrial-grade scanner to derive reference data for analyzing trueness; however, only the incisors and premolars could be captured. ${ }^{16}$

Accordingly, the purpose of this study was to evaluate the in vivo trueness and precision of full-arch scans acquired using five intraoral scanners and an industrialgrade scanner for reference scans. In addition, we aimed to compare the trueness and precision of intra-arch linear dimensions across the canines and molars. The null hypothesis was that there are no differences in the trueness and precision of full-arch scan data among different intraoral scanners.

\section{MATERIALS AND METHODS}

This prospective clinical study was approved by the Institutional Review Board of the Korea University Anam Hospital (2019AN0195). Nine healthy participants with a complete permanent dentition were recruited, and all of them provided written informed consent for participation in the study. The inclusion criteria were as follows: (1) age $\geq 19$ years; (2) complete permanent dentition with healthy periodontium; and (3) healthy general condition that would facilitate cooperation during multiple intraoral scanning procedures. The exclusion criteria were as follows: (1) missing permanent teeth, excluding third molars; (2) two or more prostheses; (3) mouth opening < $35 \mathrm{~mm}$; (4) symptoms of temporomandibular joint disorders; (4) crowding $>7 \mathrm{~mm}$; and (5) asymmetry in the arch form.

The American National Standard Institute/American Dental Association Standard (ANSI/ADA) No. 132 for the scanning accuracy of dental chairside and laboratory computer-aided design/computer-aided manufacturing
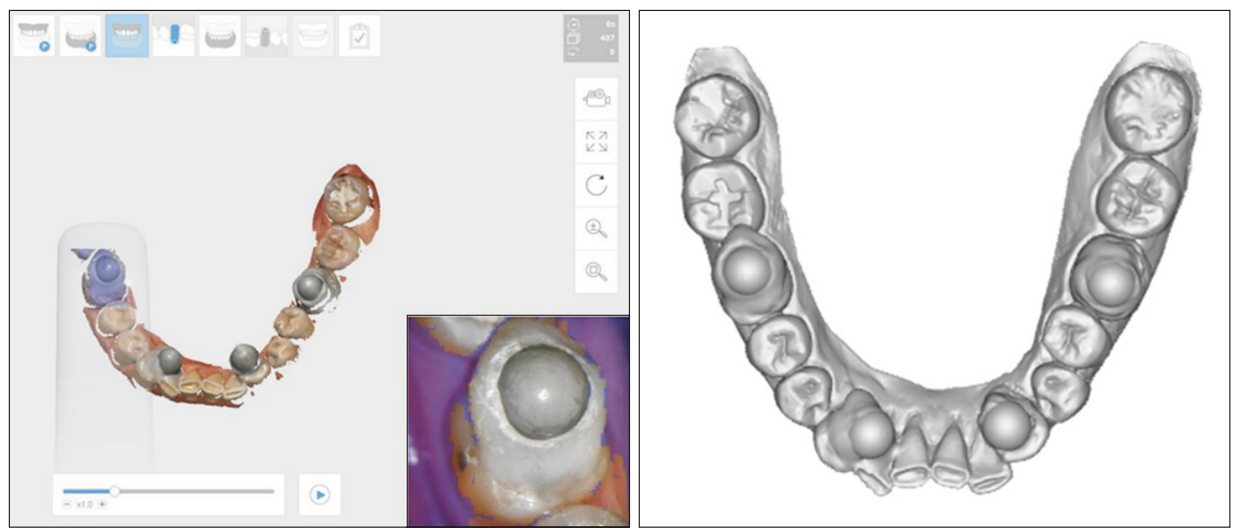

Figure 1. Placement of reference spheres for the in vivo measurement of linear distances. Four reference spheres have been attached to the lingual or palatal surface of the right and left canines and the occlusal surface of the right and left first molars. Intraoral scan data are acquired for accuracy analysis. 
systems was employed for analysis of trueness and precision. Following random selection of either the maxillary $(n=6)$ or the mandibular arch $(n=3)$, zirconium spheres (Ø 6 mm; SBB Tech Co., Gimpo, Korea) were bonded, using flowable composite resin (Filtek ${ }^{\mathrm{TM}} \mathrm{Z} 350$ XT; 3M Co., St. Paul, MN, USA), to the lingual or palatal surface of the right and left canines and the occlusal surface of the right and left first molars in the selected arch. The four spheres were references for the measurement of linear distances (Figure 1).

An industrial-grade scanner, Solutionix C500 (Medit Corp., Seoul, Korea; Table 1), was used to acquire scans of the four reference spheres. Following calibration of the scanner, each participant was positioned in the dental chair. Then, an intraoral retractor, Optragate (Ivoclar Vivadent, Schaan, Liechtenstein), was placed, the Solutionix C500 scanner was mounted on a tripod, and the four reference spheres placed in the participant's dental arches were scanned with maximum mouth opening (Figure 2).

Five commercially available intraoral scanners were

Table 1. Specifications of the reference scanner (Solutionix C500; Medit Corp., Seoul, Korea) used in the study

\begin{tabular}{ll}
\hline \multicolumn{2}{c}{ Solutionix C500 } \\
\hline Camera resolution & $2 \times 5.0 \mathrm{MP}$ \\
Point spacing & $0.028-0.157 \mathrm{~mm}$ \\
3D scanning area (FOV) & $90 / 175 / 350 / 500 \mathrm{~mm}$ \\
3D scanning principle & $\begin{array}{l}\text { Phase shifting optical } \\
\text { triangulation }\end{array}$ \\
Light source & Blue LED \\
Mount & Detachable scanner head \\
Software & ezScan 2017 (ver. 2.0.0.2)
\end{tabular}

3D, three-dimensional; FOV, field of view; MP, megapixel; LED, light emitting diode. used to scan the arches: i500 (Medit Corp.), CS3600 (Carestream Health, Rochester, NY, USA), Trios 3 (3Shape A/S, Copenhagen, Denmark), iTero (Align Technology, Inc., San Jose, CA, USA), and CEREC Omnicam (Dentsply Sirona, York, PA, USA) (Table 2). After calibration, five scans were obtained with each type of scanner for each participant, resulting in 25 scans per participant. To facilitate the scan process, three trained practitioners (M.K., M.S.K., and D.W.K.) acquired the intraoral scans using the continuous method, starting from the right molar. The intraoral and reference scans for each participant were obtained in the same visit, and subsequently, the reference spheres were removed. Scans of all participants were obtained over 2 days, and the scanners were calibrated at the beginning of each experimental day. The scan data were saved and exported in the standard tessellation language file format for analysis.

Geomagic Control X software (version 2018.1.1; Evatronix SA, Bielsko-Biala, Poland) was used to measure

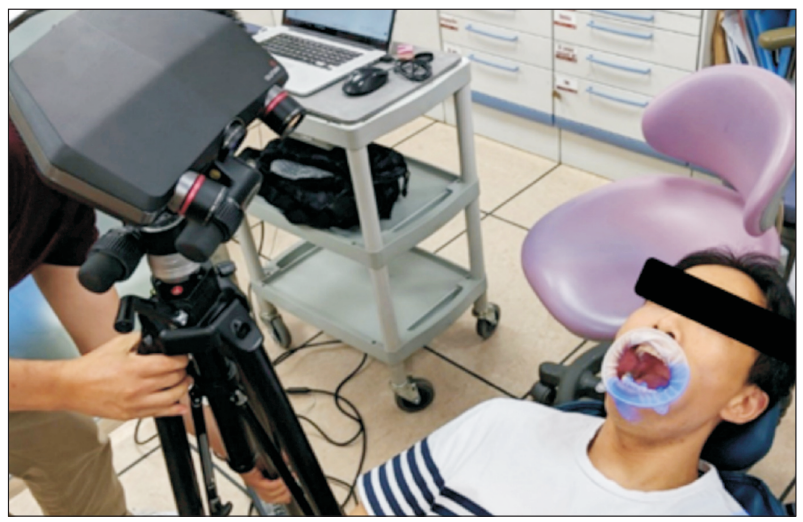

Figure 2. Reference scans for in vivo analysis of trueness. Reference scans of the four spheres are acquired using an industrial-grade scanner (Solutionix C500; Medit Corp., Seoul, Korea) for in vivo analysis of trueness.

Table 2. Specifications of the intraoral scanners used in the study

\begin{tabular}{|c|c|c|c|c|c|}
\hline System & Manufacturer & $\begin{array}{c}\text { STL export } \\
\text { software }\end{array}$ & $\begin{array}{c}\text { Software } \\
\text { version }\end{array}$ & Scanning principle & FOV $\left(\mathrm{mm}^{2}\right)$ \\
\hline $\mathrm{i} 500$ & Medit Corp., Seoul, Korea & Direct via Medit iScan & 1.1 .1 .2 & $\begin{array}{l}\text { Multi camera optical } \\
\text { triangulation }\end{array}$ & $14 \times 13$ \\
\hline CS3600 & $\begin{array}{l}\text { Carestream Health, } \\
\text { Rochester, NY, USA }\end{array}$ & Direct via CS connect & 3 & Active speed 3D video & $13 \times 13$ \\
\hline Trios 3 & $\begin{array}{l}\text { 3Shape A/S, Copenhagen, } \\
\text { Denmark }\end{array}$ & $\begin{array}{l}\text { Direct via 3Shape } \\
\text { Communicate Portal }\end{array}$ & 1.3.4.5 & $\begin{array}{l}\text { Confocal microscopy and } \\
\text { ultrafast optical scanning }\end{array}$ & $26 \times 23$ \\
\hline iTero & $\begin{array}{l}\text { Align Technology, Inc., } \\
\text { San Jose, CA, USA }\end{array}$ & Direct via OrthoCAD & 4 & $\begin{array}{l}\text { Confocal laser, } \\
\text { single image shot }\end{array}$ & $18 \times 14$ \\
\hline Omnicam & $\begin{array}{l}\text { Dentsply Sirona, York, } \\
\text { PA, USA }\end{array}$ & $\begin{array}{l}\text { Direct via Sirona } \\
\text { connect }\end{array}$ & 4.4 & $\begin{array}{l}\text { Optical triangulation and } \\
\text { confocal microscopy }\end{array}$ & $16 \times 16$ \\
\hline
\end{tabular}

STL, standard tessellation language; FOV, field of view; 3D, three-dimensional. 
the linear distances between the four reference spheres. The software automatically identifies and calculates the distance between the centers of the reference spheres by matching with pre-imputed specification data. After reorientation of the dental arches with the incisors facing up in the occlusal view, the spheres were numbered from 1 to 4 in a counterclockwise direction starting from the right lower sphere (Figure 3). Linear measurements were recorded as follows: distance 1 (between reference spheres 1 and 2), distance 2 (between reference spheres 1 and 3), distance 3 (between reference spheres 1 and 4), distance 4 (between reference spheres 2 and 3), distance 5 (between reference spheres 2 and 4), and distance 6 (between reference spheres 3 and 4; Figure 3).

The linear distances measured from the reference scan was used as true reference data. The scanning accuracy of the Solutionix C500 is reported as 7-10 $\mu \mathrm{m}$, in accordance with the system acceptance test for the scanning accuracy of optical 3D measuring systems (VDI/VDE 2634 Part 2 and 3). Trueness was evaluated by analyzing the differences in measured distances between the intraoral and reference scans. Precision was evaluated by analyzing the differences between different pairs of repeated intraoral scan data for the same participant, with 10 measurements per distance per participant.

\section{Statistical analysis}

Descriptive statistics of the trueness and precision of the linear measurements derived from the intraoral scans were obtained. Using the absolute errors, linear mixed model analysis was performed to compare the trueness and precision of the intraoral scanners according to the linear distances. Additional factors such as the practitioner who performed scanning and the arch (upper vs. lower) were considered possible confounding factors, and the significance of their effects was analyzed in the type 111 test. Through the F test, the feasibility of each statistical model with significant factors was evaluated. The Tukey-Kramer test was performed for post hoc analysis. All statistical analyses were performed using SAS software version 9.4 (SAS Institute, Cary, NC, USA). A $p$-value of $<0.05$ was considered statistically significant.

\section{RESULTS}

The study sample comprised one woman and eight men with a mean age of $34.3 \pm 8.3$ years. One participant dropped out after scanning with two scanners (iTero and Omnicam) citing personal reasons.

The boxplots of mean negative, mean positive, and mean absolute errors in terms of trueness are shown in Figures 4 and 5. The absolute mean trueness and precision values for all intraoral scanners were $76.6 \pm$ 79.3 and $56.6 \pm 52.4 \mu \mathrm{m}$, respectively (Tables 3 and 4). Omnicam showed the greatest error in terms of trueness, followed by i500, CS3600, iTero, and Trios 3 (Table 3), while CS3600 showed the greatest error in terms of precision, followed by Omnicam, i500, iTero, and Trios 3 (Table 4). Distance 3 showed the greatest error in terms of trueness, followed by distances 5, 2, 4, 1, and 6. Distance 3 also showed the greatest error in terms of precision, followed by distances 2, 5, 4, 6, and 1 (Tables 3 and 4, Figure 6).

Linear mixed model analysis revealed significant differences in trueness $(p=0.009)$ and precision $(p=$ 0.017) of some of the measured distances among the five scanners. With regard to trueness, there were sig-

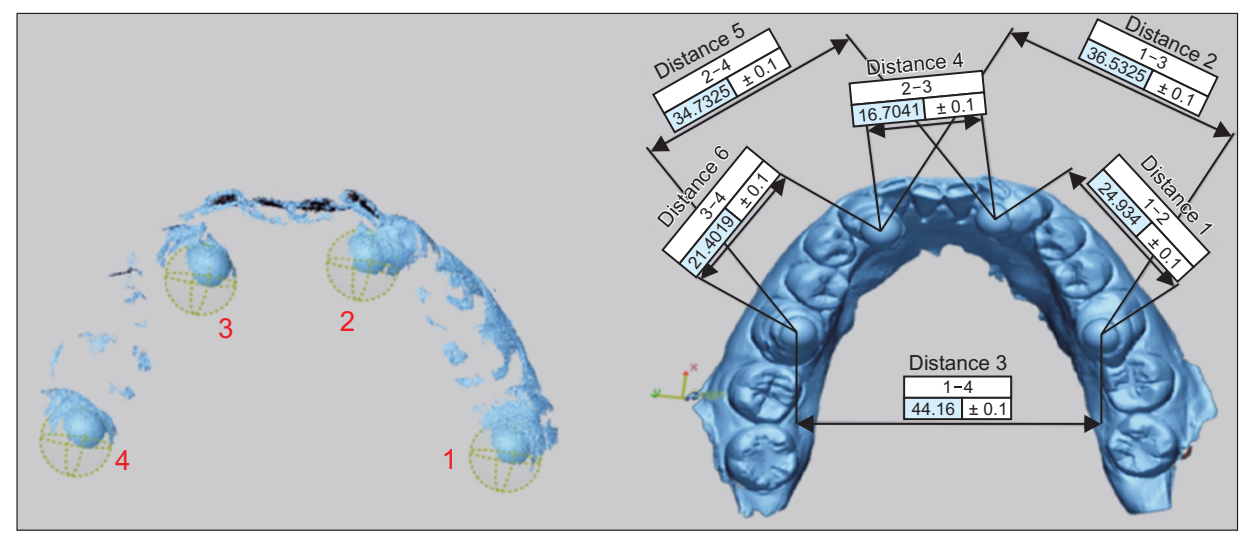

Figure 3. Measurement of linear distances. Linear distances between spheres are automatically calculated by matching with pre-imputed specification data.

Distance 1, between reference spheres 1 and 2; Distance 2, between reference spheres 1 and 3 ; Distance 3, between reference spheres 1 and 4; Distance 4, between reference spheres 2 and 3; Distance 5, between reference spheres 2 and 4; Distance 6, between reference spheres 3 and 4. 


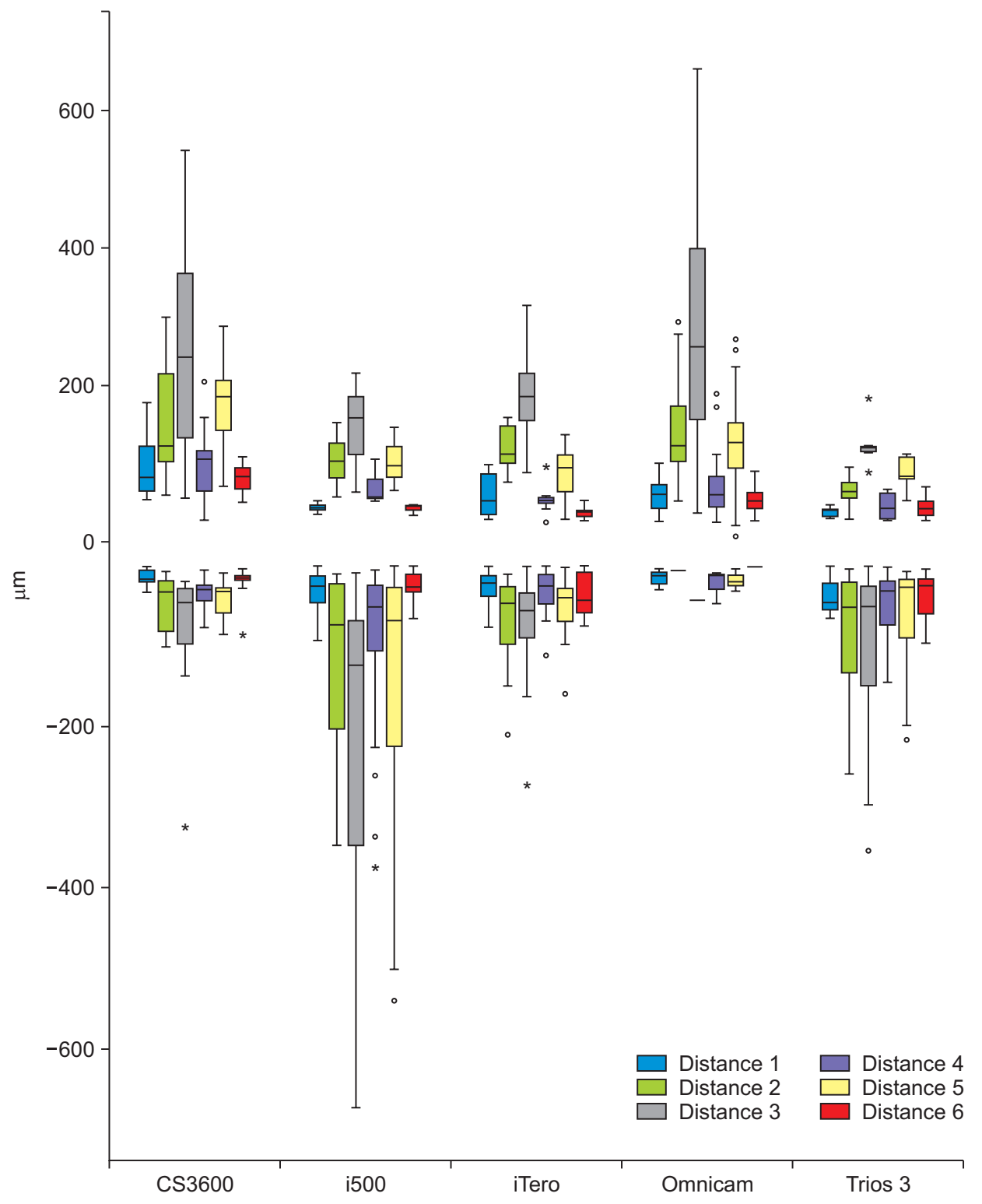

Figure 4. Mean positive and negative errors in terms of trueness according to the scanner type $(\mu \mathrm{m})$.

Distance 1 , between reference spheres 1 and 2; Distance 2, between reference spheres 1 and 3; Distance 3, between reference spheres 1 and 4 ; Distance 4 , between reference spheres 2 and 3; Distance 5, between reference spheres 2 and 4 ; Distance 6 , between reference spheres 3 and 4. nificant differences among scanners in distances 3 and 5 ( $p=0.023$ and $p=0.021$, respectively; Supplementary Table 1). With regard to precision, there were significant differences among scanners in distances 1 and $6(p=$ 0.026 and $p=0.005$, respectively; Supplementary Table 1). Other factors such as the practitioner who performed scanning and the arch (upper vs. lower) had no significant effect on the accuracy of the scan data.

According to the post hoc analysis of trueness, Omnicam showed a significantly greater error than Trios 3 for distances $3(p=0.023)$ and $5(p=0.017$; Table 5); the other scanners showed no significant differences. With regard to precision, Omnicam and CS3600 showed significantly greater errors than iTero for distance $1(p$ $=0.038$ and $p=0.042$, respectively; Table 6). For distance 6 , the precision error was significantly greater with CS3600 than with Omnicam $(p=0.021)$, iTero $(p=$ 0.047), and i500 ( $p=0.042$; Table 6).

Supplementary data is available at https://doi. org/10.4041/kjod.2021.51.2.95.

\section{DISCUSSION}

In a previous systematic review, among 2,500 studies on the accuracy of intraoral scanners, only eight in vivo studies acquired full-arch scans of patients. " From these eight studies, four only analyzed the precision of the intraoral scanner. In vivo analysis of the trueness of intraoral scanners has been challenging because it is difficult to obtain "true" reference data. The indirect method of scanning gypsum casts obtained from polyvinyl siloxane impressions involves potential errors attributed to the 


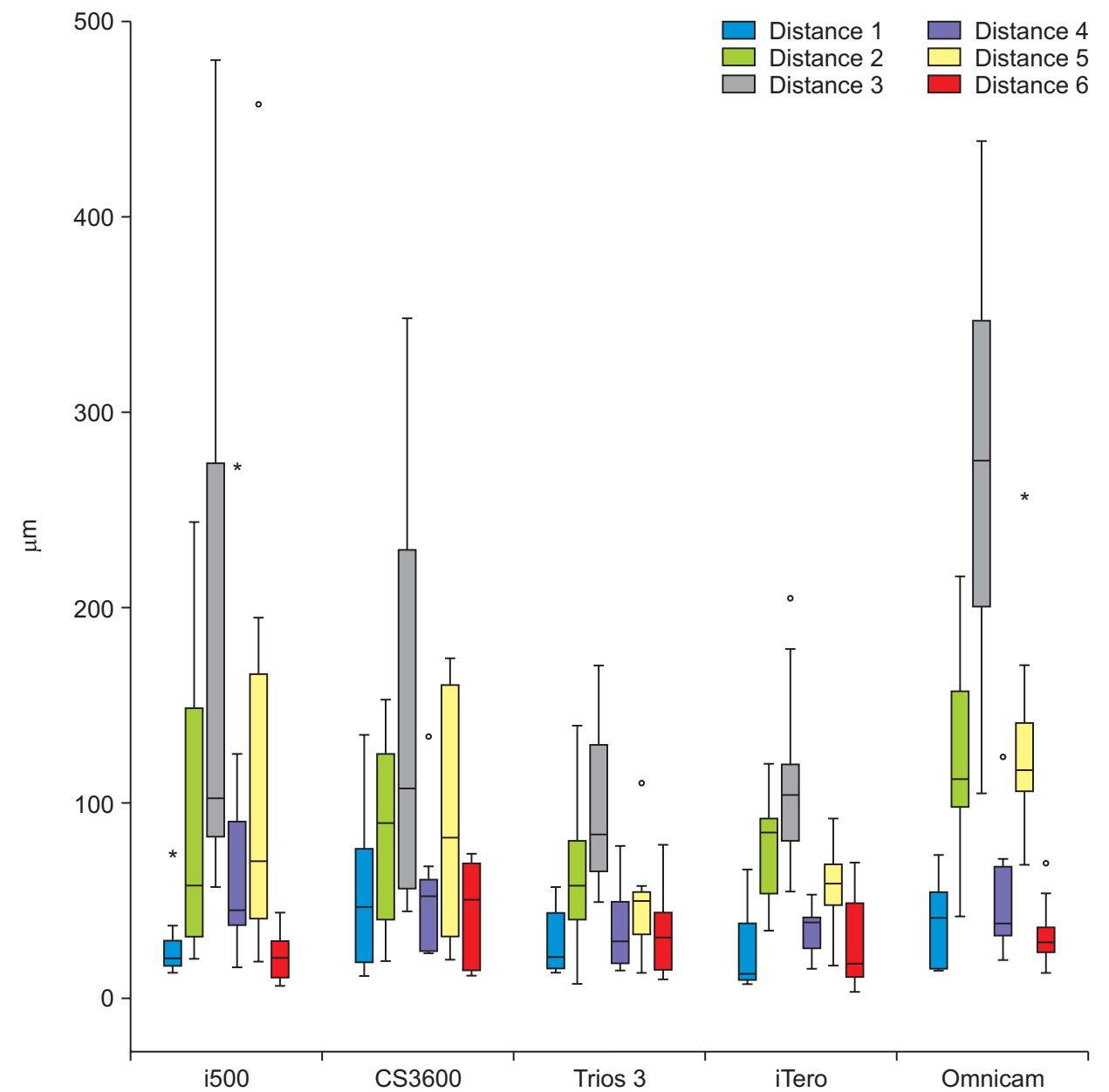

Figure 5. Mean absolute error in trueness according to the scanner type $(\mu \mathrm{m})$.

Distance 1 , between reference spheres 1 and 2; Distance 2, between reference spheres 1 and 3; Distance 3, between reference spheres 1 and 4; Distance 4 , between reference spheres 2 and 3; Distance 5, between reference spheres 2 and 4 ; Distance 6 , between reference spheres 3 and 4.

Table 3. Trueness of linear dimensions measured from intraoral scan data $(\mu \mathrm{m})$

\begin{tabular}{lccccccc}
\hline \multicolumn{1}{c}{ System } & $\mathbf{1}$ & $\mathbf{2}$ & $\mathbf{3}$ & $\mathbf{4}$ & $\mathbf{5}$ & $\mathbf{6}$ & Total \\
\hline i500 (n=8) & $27.2 \pm 20.2$ & $92.2 \pm 82.0$ & $181.8 \pm 150.3$ & $79.0 \pm 84.5$ & $128.6 \pm 145.1$ & $21.2 \pm 12.8$ & $88.3 \pm 108.8$ \\
CS3600 (n=8) & $53.6 \pm 43.7$ & $85.2 \pm 50.5$ & $147.2 \pm 112.4$ & $53.7 \pm 36.5$ & $92.6 \pm 68.4$ & $43.9 \pm 27.2$ & $79.4 \pm 69.4$ \\
Trios 3 (n=8) & $28.5 \pm 17.5$ & $62.8 \pm 40.9$ & $96.8 \pm 43.4$ & $35.3 \pm 23.9$ & $49.5 \pm 29.1$ & $33.3 \pm 22.7$ & $51.0 \pm 37.8$ \\
iTero (n=9) & $25.8 \pm 22.5$ & $74.6 \pm 28.2$ & $111.7 \pm 49.7$ & $34.7 \pm 11.8$ & $56.7 \pm 21.5$ & $27.4 \pm 23.3$ & $55.1 \pm 41.2$ \\
Omnicam (n=9) & $38.7 \pm 24.3$ & $123.9 \pm 50.4$ & $270.6 \pm 108.8$ & $51.6 \pm 32.2$ & $130.3 \pm 56.6$ & $32.9 \pm 18.0$ & $108.0 \pm 99.4$ \\
All (n= 42) & $34.6 \pm 27.8$ & $88.3 \pm 54.5$ & $163.0 \pm 115.0$ & $50.5 \pm 45.1$ & $91.6 \pm 80.4$ & $31.7 \pm 21.6$ & $76.6 \pm 79.3$ \\
\hline
\end{tabular}

Values are presented as mean \pm standard deviation.

1 , distance between reference spheres 1 and 2; 2, distance between reference spheres 1 and $3 ; 3$, distance between reference spheres 1 and 4; 4, distance between reference spheres 2 and 3; 5 , distance between reference spheres 2 and 4 ; 6 , distance between reference spheres 3 and 4 .

properties of the impression material and gypsum..$^{12,13,17}$ Kuhr et al. ${ }^{6}$ placed four metal spheres on the dental arch by using a precise metal transfer aid with predetermined distances between the balls. However, there is a possibility of errors during the process of indirect bonding. In an in vitro study, an industrial scanner showed trueness and precision errors of $5.3 \pm 1.1$ and $1.6 \pm 0.6 \mu \mathrm{m}$, re- spectively; these were smaller than the errors shown by conventional impressions and digital impressions. ${ }^{15}$ To the best of our knowledge, the first in vivo analysis of trueness using an industrial-grade scanner was reported by Nedelcu et al., ${ }^{16}$ who could only capture the reference data for the incisors and premolars. In the present study, we were able to obtain scans of the first molars by at- 
Table 4. Precision of linear dimensions measured from intraoral scan data $(\mu \mathrm{m})$

\begin{tabular}{lccccccc}
\hline \multicolumn{1}{c}{ System } & $\mathbf{1}$ & $\mathbf{2}$ & $\mathbf{3}$ & $\mathbf{4}$ & $\mathbf{5}$ & $\mathbf{6}$ & Total \\
\hline i500 $(\mathrm{n}=8)$ & $15.3 \pm 4.3$ & $62.9 \pm 38.6$ & $113.7 \pm 39.9$ & $49.9 \pm 28.2$ & $73.5 \pm 31.3$ & $12.9 \pm 4.4$ & $54.7 \pm 44.3$ \\
CS3600 (n=8) & $21.8 \pm 9.0$ & $84.9 \pm 37.3$ & $151.0 \pm 92.4$ & $45.4 \pm 22.7$ & $72.1 \pm 33.8$ & $25.7 \pm 12.3$ & $66.8 \pm 61.2$ \\
Trios 3 (n=8) & $15.5 \pm 6.5$ & $55.6 \pm 37.2$ & $98.6 \pm 61.8$ & $36.1 \pm 22.9$ & $55.9 \pm 38.8$ & $21.8 \pm 7.2$ & $47.3 \pm 43.2$ \\
iTero (n=9) & $12.3 \pm 6.2$ & $67.0 \pm 43.2$ & $116.5 \pm 55.7$ & $40.5 \pm 26.5$ & $61.2 \pm 35.3$ & $13.5 \pm 5.0$ & $51.8 \pm 48.5$ \\
Omnicam (n=9) & $20.0 \pm 5.6$ & $67.7 \pm 21.7$ & $159.0 \pm 73.7$ & $38.4 \pm 23.2$ & $73.7 \pm 45.7$ & $13.8 \pm 8.0$ & $62.1 \pm 61.0$ \\
All (n = 42) & $16.9 \pm 7.1$ & $67.6 \pm 35.7$ & $128.2 \pm 67.8$ & $41.9 \pm 24.1$ & $67.3 \pm 36.4$ & $17.4 \pm 9.1$ & $56.6 \pm 52.4$ \\
\hline
\end{tabular}

Values are presented as mean \pm standard deviation.

1 , distance between reference spheres 1 and 2; 2, distance between reference spheres 1 and 3; 3 distance between reference spheres 1 and $4 ; 4$, distance between reference spheres 2 and 3; 5, distance between reference spheres 2 and 4 ; 6 , distance between reference spheres 3 and 4 .
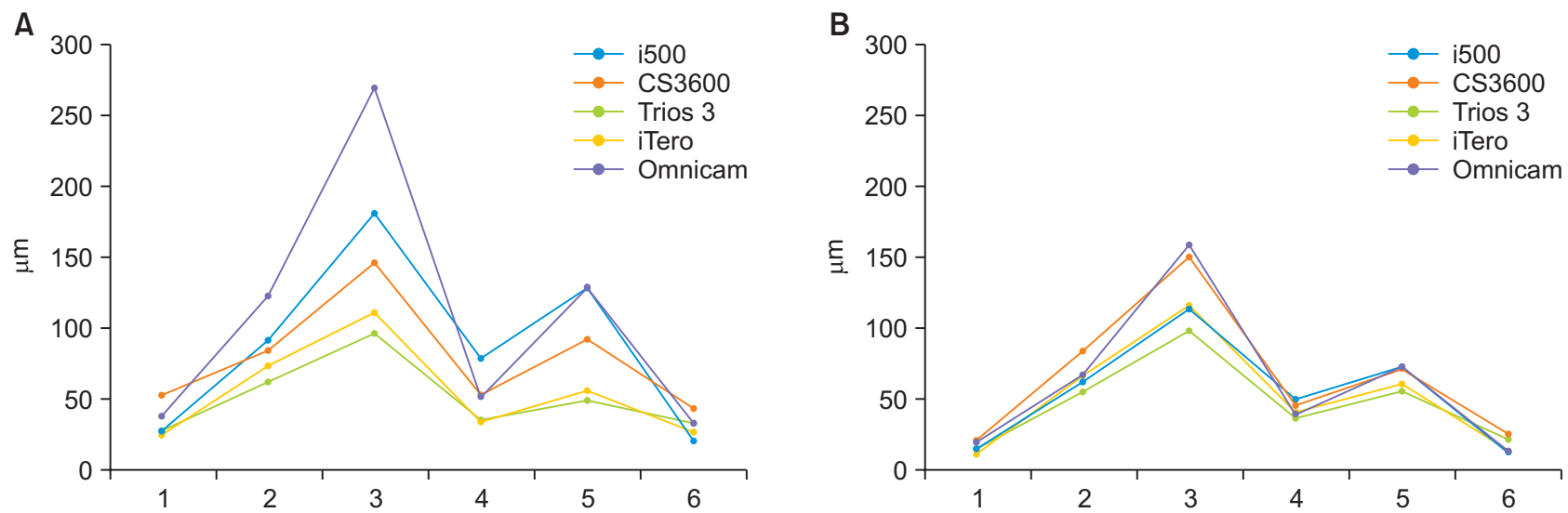

Figure 6. Mean absolute errors in terms of the trueness (A) and precision (B) of intraoral scanners according to the measured linear distances $(\mu \mathrm{m})$.

1, distance between reference spheres 1 and 2; 2, distance between reference spheres 1 and $3 ; 3$ distance between reference spheres 1 and $4 ; 4$, distance between reference spheres 2 and $3 ; 5$, distance between reference spheres 2 and $4 ; 6$, distance between reference spheres 3 and 4 .

taching reference spheres to their occlusal surface and scanning with maximum mouth opening. As the industrial scanner, Solutionix C500, provides a field of view of $175 \mathrm{~mm}$, the four reference spheres could be captured in the same frame (Figure 2).

We found significant differences in accuracy depending on the measured distances. As expected, the greatest error in terms of trueness and precision was observed in the intermolar distance, followed by the distance from the canine to the contralateral molar, the intercanine width, and the distance from the canine to the ipsilateral molar. Considering that the linear measurement made within the same quadrant is more accurate than the intercanine and intermolar distances, we could infer that a greater dimensional error occurs in the incisor area. Unlike molars and premolars, which have occlusal surfaces, incisors have sharp incisal edges, which makes it difficult to obtain a smooth scan of the incisor area. Manufac- turers suggest hovering over the labial and lingual surfaces of the incisors during the scan procedure. Because the dental arch is V-shaped, scanning errors in the incisors either increase or decrease the transverse dimension of the dental arch; thus, the intermolar distance shows the greatest error. Oh et al. ${ }^{18}$ reported that the accuracy of scan data can be affected by the orientation of the scanner head. The authors suggested that $180^{\circ}$ rotation of the scanner head should be avoided during scanning of the incisors. Another possible factor related to errors in the posterior area is that scanning of the posterior region requires more raw data to be captured than the anterior region. ${ }^{13}$ Moreover, the lighting conditions during the scan procedure show different effects on the accuracy according to the type of scanner. ${ }^{19}$

All scanners showed a large range of negative and positive dimensional errors for each measured distance. When compared with the reference data, oversized scans 
Table 5. Pairwise comparisons of trueness among scanners for distances 3 (between reference spheres 1 and 4) and 5 (between reference spheres 2 and 4 )

\begin{tabular}{|c|c|c|c|c|c|}
\hline \multirow{2}{*}{\multicolumn{2}{|c|}{ Scanners }} & \multicolumn{2}{|l|}{ Distance 3} & \multicolumn{2}{|l|}{ Distance 5} \\
\hline & & \multirow[t]{2}{*}{$\begin{array}{l}\text { Estimated difference } \\
(\mathrm{A}-\mathrm{B}) \text { in trueness }(\mu \mathrm{m})\end{array}$} & \multirow[t]{2}{*}{$p$-value } & \multirow[t]{2}{*}{$\begin{array}{l}\text { Estimated difference } \\
(\mathrm{A}-\mathrm{B}) \text { in trueness }(\mu \mathrm{m})\end{array}$} & \multirow[t]{2}{*}{$p$-value } \\
\hline A & B & & & & \\
\hline Omnicam & CS3600 & 800 & 0.130 & 590 & 0.413 \\
\hline Omnicam & iTero & 880 & 0.061 & 850 & 0.079 \\
\hline Omnicam & Trios 3 & $1,030^{*}$ & 0.023 & $1,060^{*}$ & 0.017 \\
\hline Omnicam & i500 & 580 & 0.427 & 410 & 0.747 \\
\hline CS3600 & iTero & 80 & 0.999 & 260 & 0.941 \\
\hline CS3600 & Trios 3 & 230 & 0.967 & 470 & 0.656 \\
\hline CS3600 & i500 & -220 & 0.970 & -180 & 0.986 \\
\hline iTero & Trios 3 & 150 & 0.993 & 220 & 0.970 \\
\hline iTero & i500 & -300 & 0.903 & -440 & 0.695 \\
\hline Trios 3 & i500 & -450 & 0.705 & -650 & 0.336 \\
\hline
\end{tabular}

Tukey-Kramer test was performed.

*Statistically significant.

Table 6. Pairwise comparisons of precision among scanners for distances 1 (between reference spheres 1 and 2) and 6 (between reference spheres 3 and 4)

\begin{tabular}{|c|c|c|c|c|c|}
\hline \multirow{2}{*}{\multicolumn{2}{|c|}{ Scanners }} & \multicolumn{2}{|l|}{ Distance 1} & \multicolumn{2}{|l|}{ Distance 6} \\
\hline & & \multirow[t]{2}{*}{$\begin{array}{l}\text { Estimated difference } \\
(\mathrm{A}-\mathrm{B}) \text { in precision }(\mu \mathrm{m})\end{array}$} & \multirow[t]{2}{*}{$p$-value } & \multirow[t]{2}{*}{$\begin{array}{c}\text { Estimated difference } \\
(\mathrm{A}-\mathrm{B}) \text { in precision }(\mu \mathrm{m})\end{array}$} & \multirow[t]{2}{*}{$p$-value } \\
\hline $\mathrm{A}$ & B & & & & \\
\hline Omnicam & CS3600 & -10 & 0.999 & $-723^{*}$ & 0.021 \\
\hline Omnicam & iTero & $561^{*}$ & 0.038 & -74 & 0.997 \\
\hline Omnicam & Trios 3 & 287 & 0.582 & -585 & 0.090 \\
\hline Omnicam & i500 & 276 & 0.619 & -43 & 0.999 \\
\hline CS3600 & iTero & $572^{*}$ & 0.042 & $649^{*}$ & 0.047 \\
\hline CS3600 & Trios 3 & 298 & 0.575 & 138 & 0.975 \\
\hline CS3600 & i500 & 286 & 0.611 & $680^{*}$ & 0.042 \\
\hline iTero & Trios 3 & -274 & 0.624 & -512 & 0.175 \\
\hline iTero & i500 & -286 & 0.587 & 31 & 0.999 \\
\hline Trios 3 & $\mathrm{i} 500$ & -11 & 0.999 & 543 & 0.153 \\
\hline
\end{tabular}

Tukey-Kramer test was performed.

*Statistically significant.

would have positive values while undersized scans would have negative values. If the absolute values were not taken, the positive and negative errors would add up to values closer to zero, thus underestimating the errors.

Significant differences in the trueness and precision of some of the measured distances were observed among scanners. With regard to trueness, significant differences were observed in the intermolar distance and the distance from the right canine to the left molar. With regard to precision, the distance from the canine to the molar in the same quadrant showed significant variations. Although the linear dimensions from the canine to molar within the same quadrant showed the highest precision among all measured distances, some scanners showed significant differences in performance. This significant difference in precision could be attributed to the low mean error in the linear distances measured in the same quadrant. Conversely, the precision of the 
transverse arch dimensions showed high mean errors with large standard deviations.

We found that Omnicam showed higher dimensional errors in terms of trueness than the other scanners in the intermolar dimension and the distance from the canine to the contralateral molar. Meanwhile, Omnicam and CS3600 showed greater errors in terms of precision than other scanners in the linear distance from the canine to molar in the same quadrant.

A limitation of this study was the small number of participants. Moreover, there were differences in trueness and precision among some scanners that showed borderline non-significance. A further study with a larger number of participants may lead to a better understanding of the factors associated with the accuracy of intraoral scanners. Finally, although the practitioner who performed the scanning had no significant effect, some practitioners were not familiar with certain types of intraoral scanners, which could have affected the dimensional errors.

\section{CONCLUSION}

In summary, with regard to trueness, Omnicam showed greater dimensional errors than the other scanners in the intermolar distance and the distance from the canine to the contralateral molar. With regard to precision, Omnicam and CS3600 showed greater errors than the other scanners in the linear distance from the canine to the molar in the same quadrant. Thus, the dimensional accuracy of intraoral scan data may differ significantly according to the type of scanner, with the amount of error in terms of trueness being clinically significant.

\section{CONFLICTS OF INTEREST}

No potential conflict of interest relevant to this article was reported.

\section{REFERENCES}

1. Güth JF, Keul C, Stimmelmayr M, Beuer F, Edelhoff D. Accuracy of digital models obtained by direct and indirect data capturing. Clin Oral Investig 2013; 17:1201-8.

2. Boeddinghaus M, Breloer ES, Rehmann P, Wöstmann B. Accuracy of single-tooth restorations based on intraoral digital and conventional impressions in patients. Clin Oral Investig 2015;19:2027-34.

3. Berrendero S, Salido MP, Valverde A, Ferreiroa A, Pradies G. Influence of conventional and digital intraoral impressions on the fit of CAD/CAM-fabricated all-ceramic crowns. Clin Oral Investig 2016; 20:2403-10.
4. Atieh MA, Ritter AV, Ko CC, Duqum 1. Accuracy evaluation of intraoral optical impressions: a clinical study using a reference appliance. J Prosthet Dent 2017;118:400-5.

5. Ender A, Mehl A. Influence of scanning strategies on the accuracy of digital intraoral scanning systems. Int J Comput Dent 2013;16:11-21.

6. Kuhr F, Schmidt A, Rehmann P, Wöstmann B. A new method for assessing the accuracy of full arch impressions in patients. J Dent 2016;55:68-74.

7. Rhee YK, Huh YH, Cho LR, Park CJ. Comparison of intraoral scanning and conventional impression techniques using 3-dimensional superimposition. J Adv Prosthodont 2015;7:460-7.

8. Sousa MV, Vasconcelos EC, Janson G, Garib D, Pinzan A. Accuracy and reproducibility of 3-dimensional digital model measurements. Am J Orthod Dentofacial Orthop 2012;142:269-73.

9. Lee KM. Comparison of two intraoral scanners based on three-dimensional surface analysis. Prog Orthod 2018;19:6.

10. Li H, Lyu P, Wang Y, Sun Y. Influence of object translucency on the scanning accuracy of a powderfree intraoral scanner: a laboratory study. J Prosthet Dent 2017;117:93-101.

11. Goracci C, Franchi L, Vichi A, Ferrari M. Accuracy, reliability, and efficiency of intraoral scanners for full-arch impressions: a systematic review of the clinical evidence. Eur J Orthod 2016;38:422-8.

12. Ender A, Attin T, Mehl A. In vivo precision of conventional and digital methods of obtaining complete-arch dental impressions. J Prosthet Dent 2016; 115:313-20.

13. Flügge TV, Schlager S, Nelson K, Nahles S, Metzger MC. Precision of intraoral digital dental impressions with iTero and extraoral digitization with the iTero and a model scanner. Am J Orthod Dentofacial Orthop 2013;144:471-8.

14. Grünheid T, McCarthy SD, Larson BE. Clinical use of a direct chairside oral scanner: an assessment of accuracy, time, and patient acceptance. Am J Orthod Dentofacial Orthop 2014;146:673-82.

15. Ender A, Mehl A. Accuracy of complete-arch dental impressions: a new method of measuring trueness and precision. J Prosthet Dent 2013;109:121-8.

16. Nedelcu R, Olsson P, Nyström I, Rydén J, Thor A. Accuracy and precision of 3 intraoral scanners and accuracy of conventional impressions: a novel in vivo analysis method. J Dent 2018;69:110-8.

17. Müller P, Ender A, Joda T, Katsoulis J. Impact of digital intraoral scan strategies on the impression accuracy using the TRIOS Pod scanner. Quintessence Int 2016;47:343-9.

18. Oh KC, Park JM, Moon HS. Effects of scanning 
strategy and scanner type on the accuracy of intraoral scans: a new approach for assessing the accuracy of scanned data. J Prosthodont 2020;29:51823.

19. Revilla-León M, Jiang P, Sadeghpour M, Piedra-
Cascón W, Zandinejad A, Özcan M, et al. Intraoral digital scans-part 1: influence of ambient scanning light conditions on the accuracy (trueness and precision) of different intraoral scanners. J Prosthet Dent 2020;124:372-8. 